Studies of the effect of normalized radiation fluxes on the measurement result revealed the most influential one. The value of the normalized flow $F_{0}$ was shown to have a greater effect on the relative measurement error than $\Delta F_{0}$. This allows investigating the relationship between the controlled $F_{x}$ and the normalized flow $F_{0}$. Experimental studies have confirmed that by a threefold increase in the normalized flow $F_{0}$ relative to the controlled flow $F_{x}$, it becomes possible to increase the measurement accuracy in a wide range. In particular, it was found that at the flux value $F_{0}=0.16 \cdot 10^{-3} \mathrm{~W}$, it becomes possible to measure the controlled flow in $a$ wider range $F_{x}=\left(0.16 \cdot 10^{-3} \div 0.97 \cdot 10^{-3}\right) W$ with a relative error of thousandths of a percent. The effect of the reproduction error on the measurement result under the condition of a threefold increase in the normalized flow $F_{0}$ relative to the controlled flow $F x$ is shown. It was found that an increase in the reproduction error of the normalized radiation fluxes by 1 order leads to a narrowing of the range in which the value of the relative error tends to zero. It is shown that in the absence of a threefold increase in the normalized flow $F_{0}$, an increase in the reproduction error of the normalized flows by 1 order leads to individual cases of reduction in the relative error to small-order values. The latter, by the way, applies to cases where the ratio between the normalized $F_{0}$ and controlled flow $F_{x}$, as 3 to 1 , is ensured. It is shown that the reproduction error of the dark flow does not affect the measurement result.

Thus, there is reason to believe that it is possible to expand the measurement range, in which the value of the relative error is thousandths of a percent, even for 1 measurement cycle

Keywords: redundant methods, measurement equations, accuracy increase, normalized quantities, reproduction errors of quantities
UDC 389:621.317

DOI: $10.15587 / 1729-4061.2021 .227984$

COMPUTER SIMULATION OF LOGARITHMIC TRANSFORMATION FUNCTION TO EXPAND THE RANGE OF HIGH-PRECISION MEASUREMENTS

\author{
Volodymyr Shcherban'
}

Doctor of Technical Sciences, Professor, Head of Department*

E-mail: shherban.vy@knutd.com.ua

Ganna Korogod

PhD, Associate Professor*

E-mail: 5618607@ukr.net

Oksana Kolysko

$\mathrm{PhD}$, Associate Professor*

E-mail: kolisko.oz@knutd.com.ua

Mariana Kolysko

$\mathrm{PhD}$, Associate Professor*

E-mail: Sholudko.mi@knutd.edu.ua

Yury Shcherban'

Doctor of Technical Sciences, Professor, Head of Department**

E-mail: scherban@i.ua

G a n n a $\mathbf{S}$ chut ska

Doctor of Technical Sciences, Associate Professor, Director**

E-mail: polischuka.kklp@gmail.com

*Department of Computer Science and Technology Kyiv National University of Technologies and Design Nemyrovycha-Danchenka str., 2, Kyiv, Ukraine, 01011

**Department of Light Industry Technologies

Kyiv College of Light Industry

Ivana Kudri str., 29, Kyiv, Ukraine, 01601
Received date 03.03.2021

Accepted date 15.04.2021 Published date 30.04.2021
How to Cite: Shcherban', V., Korogod, G., Kolysko, O., Kolysko, M., Shcherban', Y., Shchutska, G. (2021). Computer simulation of logarithmic transformation function to expand the range of high-precision measurements. Eastern-European Journal of Enterprise Technologies, 2 (9 (110)), 27-36. doi: https://doi.org/10.15587/1729-4061.2021.227984

\section{Introduction}

It is known that accuracy is one of the most important characteristics of measurements of any production or technological process. Therefore, adherence to the high accuracy of measurements at each stage of production is a priority, because it is a guarantee of manufacturing quality products or obtaining reliable information about the state or value of the controlled parameter [1-3]. In addition, special attention should be paid to complex production processes, where to determine the controlled quantity it is necessary to measure several quantities over their entire range and the final accuracy of measurements depends on the measurement accuracy of each quantity. Moreover, it is necessary to take into account both the instrumental error of the measuring tool itself and the methodological error of the measurement method, which also affect the final result. Therefore, scientific works, for example [4,5], are aimed at improving methods and means of measurement, as well as the possibility of targeted adjustment of parameters to improve the result. Also, in cases where the sensor transformation function is non-linear, there is a need for additional measures to linearize it over the entire range, which also affects the accuracy and cost of the measurement tool. So, the more accurately each quantity is measured, the more accurate the total measurement result will be. Therefore, there is a need to increase the accuracy of the measurement results of technological parameters, which would be comparable to the accuracy of reference standards. 
Thus, studies aimed at improving measurement accuracy in a wide range with a nonlinear transformation function, and also in the presence of an error of reproduction of normalized quantities should be considered relevant.

\section{Literature review and problem statement}

The requirements for improving accuracy in a wide range of measurements encourage the search for ways to solve them. So, in [6], the main recommendations to reduce the impact of additional errors affecting the measurement result were given and limits of their values were established. In [7], the accuracy was improved by introducing corrections that eliminate systematic errors of the measuring channel. However, this technique requires input information in the form of channel parameters, which must be determined in the recommended way. In [8], an increase in temperature measurement accuracy was achieved by reducing the methodological error from heat dissipation and high accuracy of the calibration characteristic. However, it should be noted that in this work, the temperature values of the calibration characteristic are tied to the interference minima of birefringence of the thermocouple sensor element. This means that if it is replaced, it is necessary to select identical thermocouples, which causes some difficulties in adjusting or performing additional calibration. Also, the effect of dark current was not reported. To solve this problem, [9] proposed to use a radiometric modular converter. It is shown that increased accuracy and sensitivity of measurement were due to the reduced influence of dark current, as well as compensation of natural noise by switching signals of reference and measured photodiodes and processing them appropriately. In [10], an increase in the accuracy of measurement by a square InGaAs detector was achieved by combining the infinite integral method with the Boltzmann method, having opposite error characteristics. In [11], a recursive method was proposed to reduce cross-noise, based on obtaining individual values of resistors in the array of resistive sensors. However, these works do not provide ways to expand the range for a nonlinear transformation function. To expand the range and reduce noise, [12] proposed to use programmed gain in a photodiode amplifier. In [13], an improved algorithm was proposed for this, which expands the linear measurement range without reducing measurement accuracy. The issues of increasing accuracy by increasing sensitivity were considered in [14]. In this work, the application of the phase-sensitive detection method for silicon avalanche photodiodes allowed improving the signal-to-noise ratio, as well as expanding the measurement range towards its initial values. The increase in sensitivity in [15] was achieved using an argon laser with a given power, and in [16] - using a graphene/silicon (G/Si) heterojunction photodetector. However, the nonlinearity of the photoconverter transformation function was not taken into account, which, in turn, causes a nonlinearity error or requires work on linear sections of the sensor input characteristic. To overcome this problem, an increase in sensor accuracy was achieved in [17] by correcting the nonlinear curvature by measuring the correction for the nonlinear curvature at several reference temperatures. In [18], the application of the adaptive neuro-fuzzy inference system (ANFIS) was proposed, which makes it possible to increase measurement accuracy due to a high capacity for nonlinear approximation. But the issues related to the instability of photodiode parameters, as well as the dependence on the correspondence tables for each individual sensor, remained unresolved. In [19], an increase in the accuracy of a sensor with a nonlinear function was achieved by linearizing the curve using a complementary metal oxide semiconductor.

Despite the practical significance of the results, the expansion of the range of high-precision measurements with nonlinear and unstable sensor transformation function has not been sufficiently considered.

The expediency of using redundant measurement methods for nonlinear and unstable transformation functions is confirmed by increased accuracy, as indicated in [20,21]. This is possible by applying the presented mathematical model, in which, by processing the results according to the equations of redundant measurements, the influence of the additive and multiplicative components of the measurement error is eliminated. However, these works did not investigate the influence of normalized values, as well as the influence of the reproduction error of normalized flows, on the expansion of the range of high-precision measurements.

Therefore, there is reason to believe that insufficient certainty of the impact of normalized quantities and their relationship with the controlled quantity on the expansion of the high (precision) accuracy measurement range necessitates research in this direction.

\section{The aim and objectives of the study}

The aim of the study is to determine the nature of the effect of normalized quantities on the measurement result and also to find such a relationship between the controlled and normalized quantities, which reduces the measurement error with a nonlinear transformation function in a wide range. This will significantly expand the measurement range, in which the relative error has a small-order value, i.e. will allow the entire technological process from start to finish with high (precision) accuracy.

To achieve the aim, the following objectives were set:

- to perform computer simulation of the presented mathematical model at different values of normalized quantities and with changes in the transformation function parameters within $\pm 1.0 \%$;

- to find such a relationship between the values of the controlled and the most influential normalized quantities, which provides an expansion of the measurement range with the lowest error;

- to carry out computer simulation of the effect of reproduction errors, both normalized and non-normalized quantities, on the measurement result with the found relationship between the controlled and the most influential quantities.

\section{Materials and methods of research of computer simulation of the logarithmic transformation function}

\subsection{Research materials and simulation tools}

To study the effect of the values of normalized quantities on measurement error, as well as to determine a relationship between the measured and normalized quantities, the FD307 silicon photodiode (Ukraine) was chosen as a sensor. According to the passport data, the FD307 photodiode has the following parameters: photodiode dark current $I s=0.003 \mu \mathrm{A}$, current (monochromatic) sensitivity $S_{I \lambda}=0.27 \mathrm{~A} / \mathrm{W}($ at $\lambda=0.55 \mu \mathrm{m})$. 
As a software environment, as a means for mathematical modeling and analysis of sensor behavior at different input data, Mathcad15.0 (USA) and Excel data analysis package (USA) were chosen.

\section{2. Method of research of redundant measurements}

As is known [22], the transformation function (TF) of the photodiode operating in the photovoltaic mode (load mode) has the following form:

$$
U_{R}=\frac{k T}{q} \ln \left(\frac{S_{I} F_{x}}{I_{S}}+1\right)-U_{R M},
$$

where $U_{R}$ - load voltage;

$k$ - Boltzmann constant $\left(k=1.38 \cdot 10^{-23} \mathrm{~J} / \mathrm{K}\right)$;

$T$ - photodiode temperature (ambient temperature or temperature stabilization unit);

$q$ - electron charge $\left(q=1.6 \cdot 10^{-19} \mathrm{Cl}\right)$;

$S_{I}$ - current (monochromatic) sensitivity of the photodiode;

$F_{x}$ - flux of optical radiation incident on the photodiode;

$I_{S}$ - dark current of the photodiode;

$U_{R M}$ - voltage drop across the ohmic elements of the diode.

After making the following changes: $k T / q=S_{H}^{\prime}$ (where $S_{H}^{\prime}$ - steepness of the transformation), as well as $\frac{S_{I}}{I_{S}}=\frac{1}{F_{T}}$ (where $F_{T}$ - dark flow), expression (1) takes a simplified form:

$$
U_{R}^{\prime}=S_{H}^{\prime} \ln \left(\frac{F_{x}}{F_{T}}+1\right)-U_{R M}^{\prime} .
$$

It should be noted that in the equation of quantities (2), the parameters $U_{R M}^{\prime}$ and $S_{H}^{\prime}$ are indicated with dashes, i.e. these values are not ideal and have certain deviations from their nominal values in real measurements. As a result, when using non-redundant methods, there will be a systematic error component due to the instability of the parameters of the transformation function. In addition, the nonlinear transformation function is subject to linearization, which also becomes a source of nonlinearity error, or is subject to distribution into linear sections, which narrows the operating measurement range. To solve problems of reducing the error from nonlinearity and instability of the transformation function, the redundant measurement method (RMM) was chosen as a research method.

As is known [24-26], to implement redundant measurements, a mathematical model is developed in the form of a system of equations describing time-distributed measurement cycles. The measurement cycles were determined using the following method. First, the number of variables in the presented TF is determined, on the basis of which the number of measurement cycles is formed. Then, using standard sources with normalized characteristics, normalized radiation fluxes $F_{0}$ and $\Delta F_{0}$ are formed, which are measured. Thus, in each cycle, measurements of the controlled quantity $F_{x}$, normalized radiation fluxes $F_{0}$ and $\Delta F_{0}$ or a combination thereof are carried out. As can be seen from equation (2), the logarithmic TF has 4 variables. Therefore, it is necessary to form 4 measurement cycles, which will be described by 4 equations of quantities.

We present the mathematical model as the following system of equations:

$$
\left\{\begin{array}{l}
U_{R 1}^{\prime}=S_{H}^{\prime} \ln \left(F_{0} / F_{T}+1\right)-U_{R M}^{\prime}, \\
U_{R 2}^{\prime}=S_{H}^{\prime} \ln \left(\left(F_{0}+\Delta F_{0}\right) / F_{T}+1\right)-U_{R M}^{\prime}, \\
U_{R 3}^{\prime}=S_{H}^{\prime} \ln \left(\left(F_{x}\right) / F_{T}+1\right)-U_{R M}^{\prime}, \\
U_{R 4}^{\prime}=S_{H}^{\prime} \ln \left(\left(F_{x}+\Delta F_{0}\right) / F_{T}+1\right)-U_{R M}^{\prime},
\end{array}\right.
$$

where $U_{R i}^{\prime}$ - voltage in each $i$-th $(i=(1 \div 4))$ measurement cycle.

To solve this system with respect to the controlled quantity $F_{x}$, we find the voltage differences $\left(U_{R 4}^{\prime}-U_{R 3}^{\prime}\right)$ and $\left(U_{R 2}^{\prime}-U_{R 1}^{\prime}\right)$ and express them through the parameter $S_{H}^{\prime}$. As a result, we obtain the following equation of quantities:

$$
\frac{U_{R 2}^{\prime}-U_{R 1}^{\prime}}{\ln \left(\frac{\Delta F_{0}}{F_{0}+F_{T}}+1\right)}=\frac{U_{R 4}^{\prime}-U_{R 3}^{\prime}}{\ln \left(\frac{\Delta F_{0}}{F_{x}+F_{T}}+1\right)} \text {. }
$$

As can be seen from the obtained equation (4), the value of the normalized flow $F_{0}$ is in a certain ratio to $F_{x}$. Similarly, there is a reason to take a closer look at this relationship. To do this, the equation of redundant measurements of the controlled flow should be first obtained. Therefore, solving equation (4), the following expression was obtained:

$$
\begin{aligned}
& F_{x}= \\
& =\frac{\Delta F_{0}}{\left[\left(\Delta F_{0} /\left(\left(F_{0}+F_{T}\right)+1\right)\right)^{\left(U_{R 4}^{\prime}-U_{R 3}^{\prime}\right) /\left(U_{R 2}^{\prime}-U_{R 1}^{\prime}\right)}\right]-1}-F_{T} .
\end{aligned}
$$

The obtained equation (5) does not include the parameters $U_{R M}^{\prime}$ and $S_{H}^{\prime}$, that is, the result of measuring the controlled quantity $F_{x}$ does not depend on the TF parameters and their deviations from the nominal values. Thus, the RMM helps to eliminate the systematic error component caused by the instability of the transformation function parameters, and also allow you to work on the measurement results without additional linearization of the logarithmic TF. It should be noted that this result is achieved if, during the measurement cycle, which implements the proposed 4 measurement cycles, the parameter changes remain constant.

\section{Results of computer simulation of RMM with logarithmic transformation function}

5. 1. Computer simulation to determine the effect of normalized flows on the measurement result

Based on the proposed substitution $\left(S_{I} / I_{S}=1 / F_{T}\right)$, the dark flux value $F_{T}=1.111 \times 10^{-8} \mathrm{~W}$ was obtained. The operating range of the controlled radiation flux was set within $F_{x}=\left(0.90 \times 10^{-5} \div 0.97 \times 10^{-3}\right) \mathrm{W}$. Based on equation (4), the range of the normalized flux $F_{0}$ was given within $\left(0.80 \times 10^{-5} \div 0.96 \times 10^{-3}\right) \mathrm{W}$. For computer simulation, each of these two ranges was divided into 14 steps. The studies were also performed with three values of the normalized flux $\Delta F_{0}$, which are $0.001 \times 10^{-3} \mathrm{~W}, 0.01 \times 10^{-3} \mathrm{~W}$ and $0.1 \times 10^{-3} \mathrm{~W}$, respectively. The value of the reproduction error of the normalized radiation fluxes $F_{0}$ and $\Delta F_{0}$ was $\mathrm{D}_{F}=0.1 \times 10^{-6} \mathrm{~W}$. In addition, change limits for the parameters $U_{R M}^{\prime}$ and $S_{H}^{\prime}$ lying within $\pm 1.0 \%$, were set.

As a result of computer simulation in the Mathcad15.0 environment, the following data on the relative measurement error at different values of the normalized flow $\Delta F_{0}$ were obtained, which are presented in Table 1. 
Table 1

Relative measurement errors at different values of the normalized flow $\Delta F_{0},(\%)$

\begin{tabular}{|c|c|c|c|c|c|c|c|c|c|c|c|c|c|c|}
\hline \multicolumn{15}{|c|}{$\Delta F_{0}=0.001 \times 10^{-3} \mathrm{~W}$} \\
\hline flux & $F_{01}$ & $F_{02}$ & $F_{03}$ & $F_{04}$ & $F_{05}$ & $F_{06}$ & $F_{07}$ & $F_{08}$ & $F_{09}$ & $F_{010}$ & $F_{011}$ & $F_{012}$ & $F_{013}$ & $F_{014}$ \\
\hline$F_{x 1}$ & 0.34 & 0.43 & 0.46 & 0.48 & 0.49 & 0.50 & 0.50 & 0.51 & 0.51 & 0.51 & 0.51 & 0.52 & 0.52 & 0.52 \\
\hline$F_{x 2}$ & 0.13 & 0.04 & 0.00 & 0.00 & 0.02 & 0.02 & 0.03 & 0.03 & 0.04 & 0.04 & 0.04 & 0.04 & 0.04 & 0.04 \\
\hline$F_{x 3}$ & 0.15 & 0.07 & 0.04 & 0.02 & 0.01 & 0.00 & 0.00 & 0.00 & 0.00 & 0.01 & 0.01 & 0.01 & 0.01 & 0.02 \\
\hline$F_{x 4}$ & 0.16 & 0.08 & 0.05 & 0.03 & 0.02 & 0.01 & 0.00 & 0.00 & 0.00 & 0.00 & 0.00 & 0.00 & 0.00 & 0.00 \\
\hline$F_{x 5}$ & 0.17 & 0.08 & 0.05 & 0.04 & 0.03 & 0.02 & 0.01 & 0.01 & 0.00 & 0.00 & 0.00 & 0.00 & 0.00 & 0.00 \\
\hline$F_{x 6}$ & 0.17 & 0.09 & 0.06 & 0.04 & 0.03 & 0.02 & 0.02 & 0.01 & 0.01 & 0.00 & 0.00 & 0.00 & 0.00 & 0.00 \\
\hline$F_{x 7}$ & 0.17 & 0.09 & 0.06 & 0.04 & 0.03 & 0.02 & 0.02 & 0.02 & 0.01 & 0.01 & 0.00 & 0.00 & 0.00 & 0.00 \\
\hline$F_{x 8}$ & 0.18 & 0.09 & 0.06 & 0.04 & 0.03 & 0.03 & 0.02 & 0.02 & 0.01 & 0.01 & 0.01 & 0.00 & 0.00 & 0.00 \\
\hline$F_{x 9}$ & 0.18 & 0.09 & 0.06 & 0.04 & 0.03 & 0.03 & 0.02 & 0.02 & 0.02 & 0.01 & 0.01 & 0.00 & 0.00 & 0.00 \\
\hline$F_{x 10}$ & 0.18 & 0.09 & 0.06 & 0.05 & 0.04 & 0.03 & 0.02 & 0.02 & 0.02 & 0.01 & 0.01 & 0.01 & 0.00 & 0.00 \\
\hline$F_{x 11}$ & 0.18 & 0.09 & 0.06 & 0.05 & 0.04 & 0.03 & 0.02 & 0.02 & 0.02 & 0.01 & 0.01 & 0.01 & 0.01 & 0.00 \\
\hline$F_{x 12}$ & 0.18 & 0.09 & 0.06 & 0.05 & 0.04 & 0.03 & 0.02 & 0.02 & 0.02 & 0.02 & 0.01 & 0.01 & 0.01 & 0.00 \\
\hline$F_{x 13}$ & 0.18 & 0.10 & 0.06 & 0.05 & 0.04 & 0.03 & 0.03 & 0.02 & 0.02 & 0.02 & 0.01 & 0.01 & 0.01 & 0.00 \\
\hline$F_{x 14}$ & 0.18 & 0.10 & 0.06 & 0.05 & 0.04 & 0.03 & 0.03 & 0.02 & 0.02 & 0.02 & 0.01 & 0.01 & 0.01 & 0.01 \\
\hline \multicolumn{15}{|c|}{$\Delta F_{0}=0.01 \times 10^{-3} \mathrm{~W}$} \\
\hline$F_{x 1}$ & 0.17 & 0.28 & 0.32 & 0.35 & 0.36 & 0.37 & 0.38 & 0.38 & 0.39 & 0.39 & 0.39 & 0.39 & 0.40 & 0.40 \\
\hline$F_{x 2}$ & 0.13 & 0.05 & 0.01 & 0.00 & 0.01 & 0.02 & 0.03 & 0.03 & 0.03 & 0.04 & 0.04 & 0.04 & 0.04 & 0.04 \\
\hline$F_{x 3}$ & 0.15 & 0.07 & 0.04 & 0.02 & 0.01 & 0.00 & 0.00 & 0.00 & 0.00 & 0.00 & 0.01 & 0.01 & 0.01 & 0.02 \\
\hline$F_{x 4}$ & 0.16 & 0.08 & 0.05 & 0.03 & 0.02 & 0.01 & 0.00 & 0.00 & 0.00 & 0.00 & 0.00 & 0.00 & 0.00 & 0.00 \\
\hline$F_{x 5}$ & 0.16 & 0.08 & 0.05 & 0.04 & 0.03 & 0.02 & 0.01 & 0.01 & 0.00 & 0.00 & 0.00 & 0.00 & 0.00 & 0.00 \\
\hline$F_{x 6}$ & 0.16 & 0.09 & 0.06 & 0.04 & 0.03 & 0.02 & 0.02 & 0.01 & 0.01 & 0.00 & 0.00 & 0.00 & 0.00 & 0.00 \\
\hline$F_{x 7}$ & 0.17 & 0.09 & 0.06 & 0.04 & 0.03 & 0.02 & 0.02 & 0.02 & 0.01 & 0.01 & 0.00 & 0.00 & 0.00 & 0.00 \\
\hline$F_{x 8}$ & 0.17 & 0.09 & 0.06 & 0.04 & 0.03 & 0.03 & 0.02 & 0.02 & 0.01 & 0.01 & 0.01 & 0.00 & 0.00 & 0.00 \\
\hline$F_{x 9}$ & 0.17 & 0.09 & 0.06 & 0.04 & 0.03 & 0.03 & 0.02 & 0.02 & 0.02 & 0.01 & 0.01 & 0.00 & 0.00 & 0.00 \\
\hline$F_{x 10}$ & 0.17 & 0.09 & 0.06 & 0.04 & 0.03 & 0.03 & 0.02 & 0.02 & 0.02 & 0.01 & 0.01 & 0.01 & 0.00 & 0.00 \\
\hline$F_{x 11}$ & 0.17 & 0.09 & 0.06 & 0.05 & 0.04 & 0.03 & 0.02 & 0.02 & 0.02 & 0.01 & 0.01 & 0.01 & 0.01 & 0.00 \\
\hline$F_{x 12}$ & 0.17 & 0.09 & 0.06 & 0.05 & 0.04 & 0.03 & 0.02 & 0.02 & 0.02 & 0.02 & 0.01 & 0.01 & 0.01 & 0.00 \\
\hline$F_{x 13}$ & 0.17 & 0.09 & 0.06 & 0.05 & 0.04 & 0.03 & 0.02 & 0.02 & 0.02 & 0.02 & 0.01 & 0.01 & 0.01 & 0.00 \\
\hline$F_{x 14}$ & 0.17 & 0.09 & 0.06 & 0.05 & 0.04 & 0.03 & 0.03 & 0.02 & 0.02 & 0.02 & 0.01 & 0.01 & 0.01 & 0.01 \\
\hline \multicolumn{15}{|c|}{$\Delta F_{0}=0.1 \times 10^{-3} \mathrm{~W}$} \\
\hline$F_{x 1}$ & 0.15 & 0.03 & 0.02 & 0.05 & 0.07 & 0.09 & 0.10 & 0.11 & 0.11 & 0.12 & 0.12 & 0.13 & 0.13 & 0.13 \\
\hline$F_{x 2}$ & 0.12 & 0.06 & 0.04 & 0.02 & 0.00 & 0.00 & 0.00 & 0.00 & 0.01 & 0.02 & 0.02 & 0.02 & 0.02 & 0.02 \\
\hline$F_{x 3}$ & 0.12 & 0.07 & 0.04 & 0.03 & 0.02 & 0.01 & 0.00 & 0.00 & 0.00 & 0.00 & 0.00 & 0.00 & 0.00 & 0.00 \\
\hline$F_{x 4}$ & 0.12 & 0.07 & 0.05 & 0.03 & 0.02 & 0.02 & 0.01 & 0.00 & 0.00 & 0.00 & 0.00 & 0.00 & 0.00 & 0.00 \\
\hline$F_{x 5}$ & 0.12 & 0.07 & 0.05 & 0.04 & 0.03 & 0.02 & 0.02 & 0.01 & 0.01 & 0.00 & 0.00 & 0.00 & 0.00 & 0.00 \\
\hline$F_{x 6}$ & 0.12 & 0.07 & 0.05 & 0.04 & 0.03 & 0.02 & 0.02 & 0.02 & 0.01 & 0.01 & 0.00 & 0.00 & 0.00 & 0.00 \\
\hline$F_{x 7}$ & 0.12 & 0.07 & 0.05 & 0.04 & 0.03 & 0.02 & 0.02 & 0.02 & 0.01 & 0.01 & 0.00 & 0.00 & 0.00 & 0.00 \\
\hline$F_{x 8}$ & 0.12 & 0.07 & 0.05 & 0.04 & 0.03 & 0.03 & 0.02 & 0.02 & 0.01 & 0.01 & 0.01 & 0.00 & 0.00 & 0.00 \\
\hline$F_{x 9}$ & 0.12 & 0.07 & 0.05 & 0.04 & 0.03 & 0.03 & 0.02 & 0.02 & 0.02 & 0.01 & 0.01 & 0.01 & 0.00 & 0.00 \\
\hline$F_{x 10}$ & 0.12 & 0.07 & 0.05 & 0.04 & 0.03 & 0.03 & 0.02 & 0.02 & 0.02 & 0.01 & 0.01 & 0.01 & 0.00 & 0.00 \\
\hline$F_{x 11}$ & 0.12 & 0.07 & 0.05 & 0.04 & 0.03 & 0.03 & 0.02 & 0.02 & 0.02 & 0.01 & 0.01 & 0.01 & 0.01 & 0.00 \\
\hline$F_{x 12}$ & 0.12 & 0.07 & 0.05 & 0.04 & 0.03 & 0.03 & 0.02 & 0.02 & 0.02 & 0.02 & 0.01 & 0.01 & 0.01 & 0.00 \\
\hline$F_{x 13}$ & 0.12 & 0.07 & 0.05 & 0.04 & 0.03 & 0.03 & $\begin{array}{l}0.02 \\
\end{array}$ & 0.02 & 0.02 & 0.02 & 0.01 & 0.01 & 0.01 & 0.01 \\
\hline$F_{x 14}$ & 0.12 & 0.07 & 0.05 & 0.04 & 0.03 & 0.03 & 0.02 & 0.02 & 0.02 & 0.02 & 0.01 & 0.01 & 0.01 & 0.01 \\
\hline
\end{tabular}

It should be noted that the table values of relative measurement errors are not zero, but are of small order (thousandths of a percent).

From the given data (Table 1), it can be seen that the value of the normalized flow $F_{0}$ has a greater influence on the measurement error than $\Delta F_{0}$. So, a 2-order change in the value of the normalized flow $\Delta F_{0}$ does not significantly affect the result. However, it should be noted that still the best accuracy results among the presented 3 values of $\Delta F_{0}$ were obtained at $\Delta F_{0}=0.01 \times 10^{-3} \mathrm{~W}$.
5. 2. Computer simulation to determine the relationship between the normalized and controlled flows

As can be seen from Table 1, there is a certain relationship between the controlled $F_{x}$ and normalized flow $F_{0}$. To determine it, the Excel data analysis package for a nonlinear function was used. Using the solution search option, the optimal value of $F_{0}$ was found, at which the measurement error is close to zero. Thus, the relationship between $F_{0}$ and $F_{x}$ was found, which is 3:1. Therefore, a 3-fold increase in the value of the normalized flow $F_{0}$ relative to the controlled flow $F_{x}$ 
will increase the measurement range, in which the error will be of small order (even for 1 measurement cycle).

Computer simulation was performed with preliminary data as in paragraph 5. 1, but already with a 3 -fold increase in the flow $F_{0}$ relative to the flow $F_{x}$. The study was also performed at different values of the normalized flow $\Delta F_{0}$. As a result, the following values of relative errors were obtained, which are presented in Table 2 .

Table 2

Relative measurement errors at different values of the normalized flow $\Delta F_{0},(\%)$

\begin{tabular}{|c|c|c|c|c|c|c|c|c|c|c|c|c|c|c|}
\hline \multicolumn{15}{|c|}{$\Delta F_{0}=0.001 \times 10^{-3} \mathrm{~W}$} \\
\hline flux & $F_{01}$ & $F_{02}$ & $F_{03}$ & $F_{04}$ & $F_{05}$ & $F_{06}$ & $F_{07}$ & $F_{08}$ & $F_{09}$ & $F_{010}$ & $F_{011}$ & $F_{012}$ & $F_{013}$ & $F_{014}$ \\
\hline$F_{x 1}$ & 0.47 & 0.50 & 0.51 & 0.52 & 0.52 & 0.52 & 0.52 & 0.53 & 0.53 & 0.53 & 0.53 & 0.53 & 0.53 & 0.53 \\
\hline$F_{x 2}$ & 0.00 & 0.03 & 0.04 & 0.04 & 0.05 & 0.05 & 0.05 & 0.05 & 0.05 & 0.05 & 0.05 & 0.05 & 0.05 & 0.05 \\
\hline$F_{x 3}$ & 0.03 & 0.00 & 0.00 & 0.01 & 0.02 & 0.02 & 0.02 & 0.02 & 0.02 & 0.02 & 0.03 & 0.03 & 0.03 & 0.03 \\
\hline$F_{x 4}$ & 0.04 & 0.01 & 0.00 & 0.00 & 0.00 & 0.00 & 0.01 & 0.01 & 0.01 & 0.01 & 0.02 & 0.02 & 0.02 & 0.02 \\
\hline$F_{x 5}$ & 0.05 & 0.02 & 0.00 & 0.00 & 0.00 & 0.00 & 0.00 & 0.00 & 0.00 & 0.00 & 0.00 & 0.01 & 0.01 & 0.01 \\
\hline$F_{x 6}$ & 0.05 & 0.02 & 0.00 & 0.00 & 0.00 & 0.00 & 0.00 & 0.00 & 0.00 & 0.00 & 0.00 & 0.00 & 0.00 & 0.00 \\
\hline$F_{x 7}$ & 0.05 & 0.02 & 0.01 & 0.00 & 0.00 & 0.00 & 0.00 & 0.00 & 0.00 & 0.00 & 0.00 & 0.00 & 0.00 & 0.00 \\
\hline$F_{x 8}$ & 0.05 & 0.02 & 0.01 & 0.00 & 0.00 & 0.00 & 0.00 & 0.00 & 0.00 & 0.00 & 0.00 & 0.00 & 0.00 & 0.00 \\
\hline$F_{x 9}$ & 0.05 & 0.03 & 0.01 & 0.00 & 0.00 & 0.00 & 0.00 & 0.00 & 0.00 & 0.00 & 0.00 & 0.00 & 0.00 & 0.00 \\
\hline$F_{x 10}$ & 0.05 & 0.03 & 0.02 & 0.01 & 0.00 & 0.00 & 0.00 & 0.00 & 0.00 & 0.00 & 0.00 & 0.00 & 0.00 & 0.00 \\
\hline$F_{x 11}$ & 0.06 & 0.03 & 0.02 & 0.01 & 0.00 & 0.00 & 0.00 & 0.00 & 0.00 & 0.00 & 0.00 & 0.00 & 0.00 & 0.00 \\
\hline$F_{x 12}$ & 0.06 & 0.03 & 0.02 & 0.01 & 0.00 & 0.00 & 0.00 & 0.00 & 0.00 & 0.00 & 0.00 & 0.00 & 0.00 & 0.00 \\
\hline$F_{x 13}$ & 0.06 & 0.03 & 0.02 & 0.01 & 0.00 & 0.00 & 0.00 & 0.00 & 0.00 & 0.00 & 0.00 & 0.00 & 0.00 & 0.00 \\
\hline$F_{x 14}$ & 0.06 & 0.03 & 0.02 & 0.01 & 0.00 & 0.00 & 0.00 & 0.00 & 0.00 & 0.00 & 0.00 & 0.00 & 0.00 & 0.00 \\
\hline \multicolumn{15}{|c|}{$\Delta F_{0}=0.01 \times 10^{-3} \mathrm{~W}$} \\
\hline$F_{x 1}$ & 0.33 & 0.37 & 0.39 & 0.39 & 0.40 & 0.40 & 0.41 & 0.41 & 0.41 & 0.41 & 0.41 & 0.41 & 0.41 & 0.41 \\
\hline$F_{x 2}$ & 0.00 & 0.02 & 0.03 & 0.04 & 0.04 & 0.05 & 0.05 & 0.05 & 0.05 & 0.05 & 0.05 & 0.05 & 0.05 & 0.05 \\
\hline$F_{x 3}$ & 0.03 & 0.00 & 0.00 & 0.01 & 0.02 & 0.02 & 0.02 & 0.02 & 0.02 & 0.02 & 0.02 & 0.02 & 0.03 & 0.03 \\
\hline$F_{x 4}$ & 0.04 & 0.01 & 0.00 & 0.00 & 0.00 & 0.00 & 0.01 & 0.01 & 0.01 & 0.01 & 0.01 & 0.02 & 0.02 & 0.02 \\
\hline$F_{x 5}$ & 0.05 & 0.02 & 0.00 & 0.00 & 0.00 & 0.00 & 0.00 & 0.00 & 0.00 & 0.00 & 0.00 & 0.01 & 0.01 & 0.01 \\
\hline$F_{x 6}$ & 0.05 & 0.02 & 0.01 & 0.00 & 0.00 & 0.00 & 0.00 & 0.00 & 0.00 & 0.00 & 0.00 & 0.00 & 0.00 & 0.00 \\
\hline$F_{x 7}$ & 0,05 & 0,02 & 0,01 & 0.00 & 0.00 & 0.00 & 0.00 & 0.00 & 0.00 & 0.00 & 0.00 & 0.00 & 0.00 & 0.00 \\
\hline$F_{x 8}$ & 0.05 & 0.02 & 0.01 & 0.00 & 0.00 & 0.00 & 0.00 & 0.00 & 0.00 & 0.00 & 0.00 & 0.00 & 0.00 & 0.00 \\
\hline$F_{x 9}$ & 0.05 & 0.03 & 0.01 & 0.00 & 0.00 & 0.00 & 0.00 & 0.00 & 0.00 & 0.00 & 0.00 & 0.00 & 0.00 & 0.00 \\
\hline$F_{x 10}$ & 0.05 & 0.03 & 0.02 & 0.01 & 0.00 & 0.00 & 0.00 & 0.00 & 0.00 & 0.00 & 0.00 & 0.00 & 0.00 & 0.00 \\
\hline$F_{x 11}$ & 0.05 & 0.03 & 0.02 & 0.01 & 0.00 & 0.00 & 0.00 & 0.00 & 0.00 & 0.00 & 0.00 & 0.00 & 0.00 & 0.00 \\
\hline$F_{x 12}$ & 0.06 & 0.03 & 0.02 & 0.01 & 0.00 & 0.00 & 0.00 & 0.00 & 0.00 & 0.00 & 0.00 & 0.00 & 0.00 & 0.00 \\
\hline$F_{x 13}$ & 0.06 & 0.03 & 0.02 & 0.01 & 0.00 & 0.00 & 0.00 & 0.00 & 0.00 & 0.00 & 0.00 & 0.00 & 0.00 & 0.00 \\
\hline$F_{x 14}$ & 0.06 & 0.03 & 0.02 & 0.01 & 0.00 & 0.00 & 0.00 & 0.00 & 0.00 & 0.00 & 0.00 & 0.00 & 0.00 & 0.00 \\
\hline \multicolumn{15}{|c|}{$\Delta F_{0}=0.1 \times 10^{-3} \mathrm{~W}$} \\
\hline$F_{x 1}$ & 0.03 & 0.09 & 0.11 & 0.13 & 0.14 & 0.14 & 0.15 & 0.15 & 0.15 & 0.15 & 0.15 & 0.16 & 0.16 & 0.16 \\
\hline$F_{x 2}$ & 0.03 & 0.00 & 0.01 & 0.02 & 0.03 & 0.03 & 0.03 & 0.03 & 0.03 & 0.03 & 0.04 & 0.04 & 0.04 & 0.04 \\
\hline$F_{x 3}$ & 0.04 & 0.01 & 0.00 & 0.00 & 0.00 & 0.01 & 0.01 & 0.02 & 0.02 & 0.02 & 0.02 & 0.02 & 0.03 & 0.03 \\
\hline$F_{x 4}$ & 0.04 & 0.02 & 0.00 & 0.00 & 0.00 & 0.00 & 0.00 & 0.00 & 0.00 & 0.01 & 0.01 & 0.01 & 0.02 & 0.02 \\
\hline$F_{x 5}$ & 0.04 & 0.02 & 0.00 & 0.00 & 0.00 & 0.00 & 0.00 & 0.00 & 0.00 & 0.00 & 0.00 & 0.00 & 0.00 & 0.00 \\
\hline$F_{x 6}$ & 0.04 & 0.02 & 0.01 & 0.00 & 0.00 & 0.00 & 0.00 & 0.00 & 0.00 & 0.00 & 0.00 & 0.00 & 0.00 & 0.00 \\
\hline$F_{x 7}$ & 0.05 & 0.02 & 0.01 & 0.00 & 0.00 & 0.00 & 0.00 & 0.00 & 0.00 & 0.00 & 0.00 & 0.00 & 0.00 & 0.00 \\
\hline$F_{x 8}$ & 0.05 & 0.02 & 0.01 & 0.00 & 0.00 & 0.00 & 0.00 & 0.00 & 0.00 & 0.00 & 0.00 & 0.00 & 0.00 & 0.00 \\
\hline$F_{x 9}$ & 0.05 & 0.02 & 0.01 & 0.00 & 0.00 & 0.00 & 0.00 & 0.00 & 0.00 & 0.00 & 0.00 & 0.00 & 0.00 & 0.00 \\
\hline$F_{x 10}$ & 0.05 & 0.02 & 0.02 & 0.01 & 0.00 & 0.00 & 0.00 & 0.00 & 0.00 & 0.00 & 0.00 & 0.00 & 0.00 & 0.00 \\
\hline$F_{x 11}$ & 0.05 & 0.03 & 0.02 & 0.01 & 0.00 & 0.00 & 0.00 & 0.00 & 0.00 & 0.00 & 0.00 & 0.00 & 0.00 & 0.00 \\
\hline$F_{x 12}$ & 0.05 & 0.03 & 0.02 & 0.01 & 0.00 & 0.00 & 0.00 & 0.00 & 0.00 & 0.00 & 0.00 & 0.00 & 0.00 & 0.00 \\
\hline$F_{x 13}$ & 0.05 & 0.03 & 0.02 & 0.01 & 0.00 & 0.00 & 0.00 & 0.00 & 0.00 & 0.00 & 0.00 & 0.00 & 0.00 & 0.00 \\
\hline$F_{x 14}$ & 0.05 & 0.03 & 0.02 & 0.01 & 0.00 & 0.00 & 0.00 & 0.00 & 0.00 & 0.00 & 0.00 & 0.00 & 0.00 & 0.00 \\
\hline
\end{tabular}


It should be noted that the table values of relative measurement errors are not zero, but are of small order (thousandths of a percent).

As can be seen from the table (Table 2), at the ratio of the values between the flows $F_{0}$ and $F_{x}$ of $3: 1$, it becomes possible to increase the measurement range, in which the error is of small order. It should also be noted that a 2 -order change in the value of the normalized flow $\Delta F_{0}$ does not significantly affect the measurement result. However, the optimal value was found to be $\Delta F_{0}=0.01 \times 10^{-3} \mathrm{~W}$. In this case, at flux values $F_{0}=1.06 \times 10^{-3} \mathrm{~W}$, it becomes possible to measure the controlled flow in a wider range $F_{x}=\left(0.16 \times 10^{-3} \div 0.97 \times 10^{-3}\right) \mathrm{W}$ with a relative error of thousandths of a percent.
5. 3. Computer simulation of the influence of reproduction errors with the found ratio between quantities

From the practical point of view, the influence of the increase in the reproduction error of the normalized radiation fluxes $F_{0}$ and $\Delta F_{0}$ on the measurement result is of interest. Therefore, computer simulation with increasing the reproduction error of the normalized flow by one order of magnitude from $\Delta_{F}=0.1 \times 10^{-6} \mathrm{~W}$ to $\Delta_{F}=0.1 \times 10^{-5} \mathrm{~W}$, as well as at the ratio of values between the flows $F_{0}$ and $F_{x}$ of $3: 1$, was performed. As a result, the following values of relative errors were obtained, which are presented in Table 3.

It should be noted that the table values of relative measurement errors are not zero, but are of small order (thousandths of a percent).

Table 3

Relative measurement errors at different values of the normalized flow $\Delta F_{0},(\%)$

\begin{tabular}{|c|c|c|c|c|c|c|c|c|c|c|c|c|c|c|}
\hline \multicolumn{15}{|c|}{$\Delta F_{0}=0.001 \times 10^{-3} \mathrm{~W}$} \\
\hline flux & $F_{01}$ & $F_{02}$ & $F_{03}$ & $F_{04}$ & $F_{05}$ & $F_{06}$ & $F_{07}$ & $F_{08}$ & $F_{09}$ & $F_{010}$ & $F_{011}$ & $F_{012}$ & $F_{013}$ & $F_{014}$ \\
\hline$F_{x 1}$ & 4.59 & 4.90 & 5.02 & 5.08 & 5.12 & 5.15 & 5.16 & 5.18 & 5.19 & 5.20 & 5.20 & 5.21 & 5.22 & 5.22 \\
\hline$F_{x 2}$ & 0.03 & 0.26 & 0.37 & 0.42 & 0.46 & 0.48 & 0.50 & 0.51 & 0.52 & 0.53 & 0.53 & 0.54 & 0.54 & 0.55 \\
\hline$F_{x 3}$ & 0.31 & 0.02 & 0.09 & 0.14 & 0.17 & 0.20 & 0.21 & 0.23 & 0.24 & 0.24 & 0.25 & 0.26 & 0.26 & 0.27 \\
\hline$F_{x 4}$ & 0.41 & 0.12 & 0.02 & 0.04 & 0.07 & 0.10 & 0.11 & 0.13 & 0.14 & 0.14 & 0.15 & 0.16 & 0.16 & 0.16 \\
\hline$F_{x 5}$ & 0.46 & 0.17 & 0.07 & 0.01 & 0.02 & 0.04 & 0.06 & 0.07 & 0.08 & 0.09 & 0.10 & 0.10 & 0.11 & 0.11 \\
\hline$F_{x 6}$ & 0.49 & 0.21 & 0.10 & 0.04 & 0.01 & 0.01 & 0.03 & 0.04 & 0.05 & 0.06 & 0.07 & 0.07 & 0.08 & 0.08 \\
\hline$F_{x 7}$ & 0.51 & 0.23 & 0.12 & 0.07 & 0.03 & 0.00 & 0.00 & 0.02 & 0.03 & 0.04 & 0.04 & 0.05 & 0.05 & 0.06 \\
\hline$F_{x 8}$ & 0.53 & 0.24 & 0.14 & 0.08 & 0.05 & 0.02 & 0.00 & 0.00 & 0.01 & 0.02 & 0.03 & 0.03 & 0.04 & 0.04 \\
\hline$F_{x 9}$ & 0.54 & 0.25 & 0.15 & 0.09 & 0.06 & 0.04 & 0.02 & 0.00 & 0.00 & 0.01 & 0.02 & 0.02 & 0.03 & 0.03 \\
\hline$F_{x 10}$ & 0.55 & 0.26 & 0.16 & 0.10 & 0.07 & 0.05 & 0.03 & 0.02 & 0.00 & 0.00 & 0.00 & 0.01 & 0.02 & 0.02 \\
\hline$F_{x 11}$ & 0.55 & 0.27 & 0.16 & 0.11 & 0.08 & 0.05 & 0.04 & 0.02 & 0.01 & 0.00 & 0.00 & 0.00 & 0.01 & 0.01 \\
\hline$F_{x 12}$ & 0.56 & 0.28 & 0.17 & 0.12 & 0.08 & 0.06 & 0.04 & 0.03 & 0.02 & 0.01 & 0.00 & 0.00 & 0.00 & 0.00 \\
\hline$F_{x 13}$ & 0.56 & 0.28 & 0.18 & 0.12 & 0.09 & 0.06 & 0.05 & 0.03 & 0.02 & 0.02 & 0.01 & 0.00 & 0.00 & 0.00 \\
\hline$F_{x 14}$ & 0.57 & 0.29 & 0.18 & 0.12 & 0.09 & 0.07 & 0.05 & 0.04 & 0.03 & 0.02 & 0.01 & 0.00 & 0.00 & 0.00 \\
\hline \multicolumn{15}{|c|}{$\Delta F_{0}=0.01 \times 10^{-3} \mathrm{~W}$} \\
\hline$F_{x 1}$ & 3.28 & 3.69 & 3.84 & 3.92 & 3.97 & 4.00 & 4.02 & 4.04 & 4.05 & 4.07 & 4.08 & 4.08 & 4.09 & 4.10 \\
\hline$F_{x 2}$ & 0.07 & 0.23 & 0.34 & $\begin{array}{l}0.39 \\
\end{array}$ & 0.43 & 0.45 & 0.47 & 0.48 & 0.49 & 0.50 & 0.51 & 0.51 & 0.52 & 0.52 \\
\hline$F_{x 3}$ & 0.32 & 0.03 & 0.07 & 0.13 & 0.17 & 0.19 & 0.21 & 0.22 & 0.23 & 0.24 & 0.24 & 0.25 & 0.25 & 0.26 \\
\hline$F_{x 4}$ & 0.41 & 0.13 & 0.02 & 0.03 & 0.07 & 0.09 & 0.11 & 0.12 & 0.13 & 0.14 & 0.15 & 0.15 & 0.16 & 0.16 \\
\hline$F_{x 5}$ & 0.45 & 0.18 & 0.07 & 0.02 & 0.02 & 0.04 & 0.06 & 0.07 & 0.08 & 0.09 & 0.10 & 0.10 & 0.11 & 0.11 \\
\hline$F_{x 6}$ & 0.48 & 0.21 & 0.10 & 0.05 & 0.01 & 0.01 & 0.03 & 0.04 & 0.05 & 0.06 & 0.06 & 0.07 & 0.07 & 0.08 \\
\hline$F_{x 7}$ & 0.50 & 0,23 & 0.12 & 0.07 & 0.03 & 0.01 & 0.00 & 0.02 & 0.03 & 0.04 & 0.04 & 0.05 & 0.05 & 0.06 \\
\hline$F_{x 8}$ & 0.52 & 0.24 & 0.14 & 0.08 & 0.05 & 0.03 & 0.00 & 0.00 & 0.01 & 0.02 & 0.03 & 0.03 & 0.04 & 0.04 \\
\hline$F_{x 9}$ & 0.53 & 0.25 & 0.15 & 0.09 & 0.06 & 0.04 & 0.02 & 0.00 & 0.00 & 0.01 & 0.02 & 0.02 & 0.03 & 0.03 \\
\hline$F_{x 10}$ & 0.54 & 0.26 & 0.16 & 0.10 & 0.07 & 0.05 & 0.03 & 0.02 & 0.00 & 0.00 & 0.00 & 0.01 & 0.02 & 0.02 \\
\hline$F_{x 11}$ & 0.54 & 0.27 & 0.16 & 0.11 & 0.08 & 0.05 & 0.04 & 0.02 & 0.01 & 0.00 & 0.00 & 0.00 & 0.01 & 0.01 \\
\hline$F_{x 12}$ & 0.55 & 0.27 & 0.17 & 0.12 & 0.08 & 0.06 & 0.04 & 0.03 & 0.02 & 0.01 & 0.00 & 0.00 & 0.00 & 0.00 \\
\hline$F_{x 13}$ & 0.55 & 0.28 & 0.17 & 0.12 & 0.09 & 0.06 & 0.05 & 0.03 & 0.02 & 0.02 & 0.01 & 0.00 & 0.00 & 0.00 \\
\hline$F_{x 14}$ & 0.56 & 0.28 & 0.18 & 0.12 & 0.09 & 0.07 & 0.05 & 0.04 & 0.03 & 0.02 & 0.01 & 0.00 & 0.00 & 0.00 \\
\hline \multicolumn{15}{|c|}{$\Delta F_{0}=0.1 \times 10^{-3} \mathrm{~W}$} \\
\hline$F_{x 1}$ & 0.34 & 0.90 & 1.14 & 1.27 & 1.35 & 1.41 & 1.45 & 1.48 & 1.51 & 1.53 & 1.55 & 1.55 & 1.58 & 1.58 \\
\hline$F_{x 2}$ & 0.29 & 0.01 & 0.14 & 0.21 & 0.25 & 0.28 & 0.30 & 0.32 & 0.33 & 0.34 & 0.35 & 0.36 & 0.36 & 0.37 \\
\hline$F_{x 3}$ & 0.37 & 0.11 & 0.00 & 0.06 & 0.10 & 0.12 & 0.14 & 0.16 & 0.17 & 0.18 & 0.19 & 0.19 & 0.20 & 0.21 \\
\hline$F_{x 4}$ & 0.41 & 0.16 & 0.06 & 0.00 & 0.03 & 0.06 & 0.07 & 0.09 & 0.10 & 0.11 & 0.11 & 0.12 & 0.13 & 0.14 \\
\hline$F_{x 5}$ & 0.43 & 0.19 & 0.10 & 0.04 & 0.00 & 0.02 & 0.04 & 0.05 & 0.06 & 0.07 & 0.07 & 0.08 & 0.09 & 0.10 \\
\hline$F_{x 6}$ & 0.45 & 0.21 & 0.12 & 0.06 & 0.03 & 0.00 & 0.01 & 0.02 & 0.03 & 0.04 & 0.05 & 0.05 & 0.06 & 0.07 \\
\hline$F_{x 7}$ & 0.45 & 0.23 & 0.13 & 0.08 & 0.05 & 0.02 & 0.00 & 0.00 & 0.02 & 0.03 & 0.03 & 0.04 & 0.05 & 0.06 \\
\hline$F_{x 8}$ & 0.46 & 0.24 & 0.14 & 0.09 & 0.06 & 0.03 & 0.02 & 0.00 & 0.00 & 0.01 & 0.02 & 0.03 & 0.05 & 0.08 \\
\hline$F_{x 9}$ & 0.47 & 0.24 & 0.15 & 0.01 & 0.07 & 0.04 & 0.03 & 0.01 & 0.00 & 0.00 & 0.00 & 0.01 & 0.02 & 0.02 \\
\hline$F_{x 10}$ & 0.47 & 0.25 & 0.16 & 0.11 & 0.07 & 0.05 & 0.03 & 0.02 & 0.01 & 0.00 & 0.00 & 0.00 & 0.01 & 0.02 \\
\hline$F_{x 11}$ & 0.47 & 0.25 & 0.16 & 0.11 & 0.08 & 0.06 & 0.04 & 0.03 & 0.02 & 0.01 & 0.00 & 0.00 & 0.00 & 0.00 \\
\hline$F_{x 12}$ & 0.48 & 0.26 & 0.17 & 0.12 & 0.08 & 0.06 & 0.05 & 0.03 & 0.02 & 0.02 & 0.00 & 0.00 & 0.00 & 0.00 \\
\hline$F_{x 13}$ & 0.48 & 0.26 & 0.17 & 0.12 & 0.09 & 0.07 & 0.05 & 0.04 & 0.03 & 0.02 & 0.01 & 0.00 & 0.00 & 0.00 \\
\hline$F_{x 14}$ & 0.48 & 0.26 & 0.17 & 0.12 & 0.09 & 0.07 & 0.05 & 0.04 & 0.03 & 0.02 & 0.02 & 0.00 & 0.00 & 0.00 \\
\hline
\end{tabular}


As can be seen from the table (Table 3), an increase in the reproduction error of the normalized radiation fluxes leads to a narrowing of the range in which the relative measurement error of small order is provided. In this case, as in the previous cases, the value of the normalized flow $\Delta F_{0}$ is optimum, which is $0.1 \times 10^{-3} \mathrm{~W}$.

We considered the case with no 3 -fold increase in the normalized flow $F_{0}$, but the reproduction error of the normalized flows was increased by 1 order. Since the value of the normalized flow $\Delta F_{0}$ has little effect on the result, the value of the flow $\Delta F_{0}$ was set to $0.01 \mathrm{~mW}$. The results of such simulation are presented in Table 4.

Relative measurement errors at $\Delta F_{0}=0.01 \mathrm{~mW},(\%)$

\begin{tabular}{|c|c|c|c|c|c|c|c|c|c|c|c|c|c|c|}
\hline flux & $F_{01}$ & $F_{02}$ & $F_{03}$ & $F_{04}$ & $F_{05}$ & $F_{06}$ & $F_{07}$ & $F_{08}$ & $F_{09}$ & $F_{010}$ & $F_{011}$ & $F_{012}$ & $F_{013}$ & $F_{014}$ \\
\hline$F_{x 1}$ & 1.67 & 2.77 & 3.20 & 3.43 & 3.57 & 3.67 & 3.74 & 3.79 & 3.84 & 3.87 & 3.90 & 3.92 & 3.94 & 3.96 \\
\hline$F_{x 2}$ & 1.25 & 0.45 & 0.13 & 0.03 & 0.14 & 0.21 & 0.26 & 0.30 & 0.33 & 0.35 & 0.37 & 0.39 & 0.40 & 0.42 \\
\hline$F_{x 3}$ & 1.46 & 0.69 & 0.38 & 0.22 & 0.12 & 0.05 & $\mathbf{0 . 0 0}$ & 0.04 & 0.07 & 0.09 & 0.11 & 0.13 & 0.14 & 0.15 \\
\hline$F_{x 4}$ & 1.54 & 0.77 & 0.47 & 0.31 & 0.21 & 0.15 & 0.10 & 0.06 & 0.03 & $\mathbf{0 . 0 0}$ & 0.01 & 0.03 & 0.04 & 0.06 \\
\hline$F_{x 5}$ & 1.58 & 0.82 & 0.52 & 0.36 & 0.26 & 0.19 & 0.15 & 0.11 & 0.08 & 0.06 & 0.04 & 0.02 & $\mathbf{0 . 0 0}$ & $\mathbf{0 . 0 0}$ \\
\hline$F_{x 6}$ & 1.61 & 0.85 & 0.55 & 0.39 & 0.29 & 0.23 & 0.18 & 0.14 & 0.11 & 0.09 & 0.07 & 0.05 & 0.04 & 0.03 \\
\hline$F_{x 7}$ & 1.63 & 0.87 & 0.57 & 0.41 & 0.31 & 0.25 & 0.20 & 0.16 & 0.13 & 0.11 & 0.09 & 0.07 & 0.06 & 0.05 \\
\hline$F_{x 8}$ & 1.64 & 0.88 & 0.58 & 0.43 & 0.33 & 0.26 & 0.21 & 0.17 & 0.15 & 0.12 & 0.10 & 0.09 & 0.07 & 0.06 \\
\hline$F_{x 9}$ & 1.65 & 0.89 & 0.59 & 0.44 & 0.34 & 0.27 & 0.22 & 0.19 & 0.16 & 0.13 & 0.11 & 0.10 & 0.08 & 0.07 \\
\hline$F_{x 10}$ & 1.66 & 0.90 & 0.60 & 0.45 & 0.35 & 0.28 & 0.23 & 0.19 & 0.17 & 0.14 & 0.12 & 0.11 & 0.09 & 0.08 \\
\hline$F_{x 11}$ & 1.66 & 0.91 & 0.61 & 0.45 & 0.35 & 0.29 & 0.24 & 0.20 & 0.17 & 0.15 & 0.13 & 0.11 & 0.10 & 0.09 \\
\hline$F_{x 12}$ & 1.67 & 0.91 & 0.62 & 0.46 & 0.36 & 0.29 & 0.24 & 0.21 & 0.18 & 0.16 & 0.14 & 0.12 & 0.11 & 0.09 \\
\hline$F_{x 13}$ & 1.67 & 0.92 & 0.62 & 0.46 & 0.36 & 0.30 & 0.25 & 0.21 & 0.18 & 0.16 & 0.14 & 0.13 & 0.11 & 0.10 \\
\hline$F_{x 14}$ & 1.67 & 0.92 & 0.62 & 0.47 & 0.37 & 0.30 & 0.25 & 0.22 & 0.19 & 0.16 & 0.15 & 0.13 & 0.12 & 0.10 \\
\hline
\end{tabular}

It should be noted that the table values of relative measurement errors are not zero, but are of small order (thousandths of a percent).

Thus, without a 3-fold increase in the normalized flow $F_{0}$, an increase in the reproduction error of the normalized flows by 1 order leads only to individual cases of reduction of the relative error to small-order values (Table 4). Moreover, this is manifested when there is a ratio between $F_{0}$ and $F_{x}$, as 3 to 1 . However, in this case, it is necessary to know the current (predicted) value of the controlled flow, which is not always known.

Further increase in the reproduction error of normalized flows by 2 orders of magnitude from $\Delta_{F}=0.1 \times 10^{-6} \mathrm{~W}$ to $\Delta_{F}=0.1 \times 10^{-4} \mathrm{~W}$ leads to a significant deterioration in results, especially at small values of the controlled quantity. In addition, the range becomes even narrower and moves to the range of high values of the controlled and normalized quantities. However, the best in this case is the value of the normalized flow $\Delta F_{0}$, which is $0.01 \times 10^{-3} \mathrm{~W}$, which provides a small-order relative measurement error at a ratio of values $F_{0}=\left(2.08 \times 10^{-3} \div 2.89 \times 10^{-3}\right) \mathrm{W}$ and the corresponding values $F_{x}=\left(0.67 \times 10^{-3} \div 0.97 \times 10^{-3}\right) \mathrm{W}$.

In the study, we considered the case of a simultaneous increase of the reproduction error of normalized flows by 1 order and an increase in the reproduction error of the dark flow $F_{T}$ (within $\pm 10 \%$ ). Computer simulation showed that the result is not affected by the reproduction error of the dark stream, i.e. the results remained as in Table 3.

\section{Discussion of the results of computer simulation of the effect of the ratio between the flows on the measurement result}

When studying the effect of normalized quantities on the measurement result, as follows from the results (Table 1), the value of the normalized flow $F_{0}$ has a greater influence than $\Delta F_{0}$. This is due to the equation of quantities (4), where the value of the normalized flow $F_{0}$ is in a certain ratio to $F_{x}$. So, the change in the value of the normalized flow $F_{0}$ leads to more significant changes in the relative error values. Instead, a 2-order change in the value of the normalized flow

Table $4 \Delta F_{0}$ does not significantly affect the result. Obviously, such a mechanism of influence of the value of the normalized flow $F_{0}$ is the factor regulating the process, which makes it possible to influence the relative measurement error. This does not differ from the practical data presented in $[7,9,17]$, the authors of which also associate the influence of certain channel parameters (dark current, introduction of corrections) on measurement accuracy.

To prove the statement about the criteria for the effect of the value of the normalized flow $F_{0}$ on the range of high-precision measurements, it must be carefully studied. When comparing the obtained values of relative errors, a certain relationship was found between the controlled $F_{x}$ and normalized value $F_{0}$, at which this error was close to zero. Thus, at flux values $F_{0}=0.56 \times 10^{-3} \mathrm{~W}$ and $\Delta F_{0}=0.1 \times 10^{-3} \mathrm{~W}$, it becomes possible to measure the controlled flow in a wider range $F_{x}=\left(0.08 \times 10^{-3} \div 0.23 \times 10^{-3}\right) \mathrm{W}$ with a relative error of thousandths of a percent. With the increased value of the normalized flow $F_{0}$, there was an expansion of the range in which the relative error is thousandths of a percent. Therefore, to identify the relationship between the flows $F_{0}$ and $F_{x}$, in which there is an increase in the measurement range of the controlled quantity with high accuracy, computer analysis was performed. As a result of the analysis, using the solution search option, it was found that the ratio between the normalized flow $F_{0}$ and the controlled quantity $F_{x}$ should be 3 to 1 . Thus, compliance with the ratio between the normalized $F_{0}$ and controlled quantity $F_{x}$ provides a high accuracy result in a wide range of values. This is evidenced by the results of studies shown in Table 2 . For example, at flux values $F_{0}=1.06 \times 10^{-3} \mathrm{~W}$ and $\Delta F_{0}=0.1 \times 10^{-3} \mathrm{~W}$, it becomes possible to measure the controlled flow in a wider range $F_{x}=\left(0.16 \times 10^{-3} \div 0.97 \times 10^{-3}\right) \mathrm{W}$ with a relative error of thousandths of a percent. That is, the ratio between the normalized flow $F_{0}$ and the controlled quantity $F_{x}$ of 3 to 1 allows expanding the measurement range, in which the relative error has a small-order value more than 5 times. This means that taking into account this ratio between the normalized and controlled flows opens the possibility for effective control over the range of high-precision measurements. This also does not differ from the conclusions of [7, 17], the authors of which also associate the increase in measurement accuracy with compliance with the corrections to the parameters. 
But, at the same time, the measurement error is affected by the methodological error. In this sense, of particular interest is the influence of the proposed relationship between the normalized and controlled flows on the methodological error. This is due to the fact that the reproduction error of normalized flows is a component of the methodological error of the proposed redundant measurement method. The studies were performed with an increase in this error by 1 and 2 orders of magnitude. It was found that increasing the error by 1 order when increasing the normalized flow $F_{0}$ three times relative to $F_{x}$ leads to a narrowing of the measurement range, in which the value of the relative error tends to zero (Table 3 ). In addition, at the initial values of the controlled quantity, the relative error takes unacceptable values. But, at the same time, if the normalized flow $F_{0}$ is not increased three times relative to $F_{x}$, this will lead only to individual cases of reduction of the relative error to small-order values (Table 4). It should be noted that in this case, there will be a ratio between $F_{0}$ and $F_{x}$ as 3 to 1 . However, this requires knowing the current value of the controlled flow, which is not always known. Thus, the results show that increasing the normalized flow $F_{0}$ three times relative to $F_{x}$ is appropriate. A further increase in the reproduction error by 2 orders of magnitude becomes acceptable only in a very narrow range of small relative error values and at large values of the controlled quantity. This is justified by the fact that the reproduction error of the normalized flow, in this case, becomes commensurate in value with the controlled flow $F_{x}$ (especially at small values of the flow $F_{x}$ ). Thus, the results, in contrast to the results in [8], indicate the influence of methodological error (reproduction error of redundant flows) not only on the measurement accuracy, but also on the measuring range.

It should be noted that measurement accuracy is also affected by the dark current, as shown in [9]. Therefore, the study of the influence of the reproduction error of the dark stream is of scientific interest. Computer simulation was performed with the reproduction error of the dark flux $F_{T}$ within $\pm 10 \%$. The study showed that, when the ratio between the flows is found, as well as when the reproduction error of normalized flows is increased by 1 order, the reproduction error of the dark stream does not affect the measurement result. This is obviously due to the small value of the dark stream.

Thus, with the ratio of the normalized $F_{0}$ to the controlled flow $F_{x}$ of 3 to 1, it becomes possible to increase the measurement range, in which the relative error is thousandths of a percent.

Such conclusions can be considered expedient from the practical point of view as allow substantiating compliance with the ratio between the normalized $F_{0}$ and the controlled quantity $F_{x}$ as 3 to 1 , at which it becomes possible to conduct technological processes with high accuracy. In this case, it becomes possible to significantly expand the measurement range, in which the relative error is of small order (thousandths of a percent), i.e. allows carrying out the entire technological process from start to finish with high accuracy. However, such an increase in the normalized flux $F_{0}$ requires the high power of the calibrated radiation source. But in cases where the current (or predicted) value of the controlled flow is known, it is not necessary to increase the normalized flow $F_{0}$ by 3 times. In this case, it is recommended only to adhere to the ratio between $F_{0}$ and $F_{x}$ as 3 to 1 , even with the reproduction error of normalized streams increased by
1 order. This makes allows reducing the power and accuracy requirements of the calibrated radiation source.

From a theoretical point of view, the results suggest that when using the RMM, the flow $F_{0}$ should be considered the weight parameter between the normalized flows $\Delta F_{0}$ and $F_{0}$. Thus, the ratio between the normalized $F_{0}$ and the controlled quantity $F_{x}$, as 3 to 1 , can increase the measurement accuracy, as well as expand the measurement range, in which the relative error is thousandths of a percent. This is one of the advantages of applying the found relationship between the flows. However, it should be noted that such high accuracy results are obtained in the case when the parameter changes remain constant during the measurement cycles. In addition, the RMM, like any other method, has a methodological error due to the reproduction error of normalized flows. Thus, increasing the reproduction error of normalized streams leads to a narrowing of the range in which the relative error is of small order. However, it should be noted that increasing the reproduction error of the dark stream, in this case, does not affect the measurement result. Therefore, it remains acceptable when the reproduction errors of normalized flows remain as small as possible. The inability to eliminate these restrictions in this study gives rise to a potentially interesting direction for further research.

\section{Conclusions}

1. Computer simulation of the presented mathematical models at different values of normalized radiation fluxes with changes in the parameters of the transformation function within $\pm 1.0 \%$ was performed. The studies have shown that the flow $F_{0}$ has the greatest influence among the normalized flows. This is manifested in the fact that the value of the flux $F_{0}$ has a greater influence on the measurement error among the normalized flows. Due to this, it can be argued that by controlling the value of the normalized flow $F_{0}$, it is possible to expand the measurement range, in which the relative error is a small-order value (thousandths of a percent).

2. The relationship between the values of the controlled flow and the most influential normalized one was found, which provides the smallest error in a wide range of measurements. Thanks to obtained ratio of values between the flows $F_{0}$ and $F_{x}$ of 3:1, it becomes possible to increase the measurement range, in which the relative error is of small order. For example, at flow values $\Delta F_{0}=0.1 \times 10^{-3} \mathrm{~W}$ and $F_{0}=1.06 \times 10^{-3} \mathrm{~W}$ (corresponding to step 5), it becomes possible to measure the controlled flow in a wider range $F_{x}=\left(0.16 \times 10^{-3} \div 0.97 \times 10^{-3}\right) \mathrm{W}$ with a relative error of thousandths of a percent. Compared to the case where this ratio is not used and at $\Delta F_{0}=0.1 \times 10^{-3} \mathrm{~W}$ and $F_{0}=0.35 \times 10^{-3} \mathrm{~W}$ (corresponding to step 5), the value will be only $F_{x}=0.08 \times 10^{-3} \mathrm{~W}$. This indicates the possibility of increasing the accuracy in a wide range by applying the given ratio between the flows $F_{0}$ and $F_{x}$. Thus, the accuracy comparable with the accuracy of the reference standards was obtained.

3. Computer simulation of the effect of reproduction error on the measurement result under the condition of ensuring the found ratio between the controlled and the most influential quantities was carried out. It was found that increasing the reproduction error of normalized radiation fluxes by 1 order leads to a narrowing of the range, which provides the relative measurement error of small order. This 
narrowing is especially evident when the ratio between the flows $F_{0}$ and $F_{x}$, as 3 to 1 , is not applied (in this case, knowing the current value of the controlled flow is required). Thus, to reduce the effect of the reproduction error of normalized flows by 1 order, the implementation of the ratio between the flows $F_{0}$ and $F_{x}$, as 3 to 1 , is desirable. The results of the study showed that the requirements for compliance with the reproduction error of normalized flows as small as possible still remain high. Computer simulation of the effect of dark stream reproduction error was performed. It was found that the reproduction error of the dark stream does not affect the measurement result.

\section{References}

1. Pronin, A. N., Sapozhnikova, K. V., Taymanov, R. E. (2015). Reliability of measurement information in control systems. Problems and their solution. T-Comm, 9 (3), 32-37. Available at: https://cyberleninka.ru/article/n/dostovernost-izmeritelnoy-informatsiiv-sistemah-upravleniya-problemy-i-resheniya/viewer

2. Shcherban', V., Melnyk, G., Sholudko, M., Kolysko, O., Kalashnyk, V. (2018). Yarn tension while knitting textile fabric. Fibres and Textiles, 3, 74-83. Available at: http://vat.ft.tul.cz/2018/3/VaT_2018_3_12.pdf

3. Shcherban', V., Kolysko, O., Melnyk, G., Sholudko, M., Shcherban', Y., Shchutska, G. (2020). Determining tension of yarns when interacting with guides and operative parts of textile machinery having the torus form. Fibres and Textiles, 4, 87-95. Available at: http://vat.ft.tul.cz/2020/4/VaT_2020_4_12.pdf

4. Shcherban', V., Melnyk, G., Sholudko, M., Kolysko, O., Kalashnyk, V. (2019). Improvement of structure and technology of manufacture of multilayer technical fabric. Fibres and Textiles, 2, 54-63. Available at: http://vat.ft.tul.cz/2019/2/VaT_2019_2_10.pdf

5. Shcherban', V., Makarenko, J., Melnyk, G., Shcherban', Y., Petko, A., Kirichenko, A. (2019). Effect of the yarn structure on the tension degree when interacting with high-curved guides. Fibres and Textiles, 4, 59-68. Available at: http://vat.ft.tul.cz/2019/4/ VaT_2019_4_8.pdf

6. Hodovanyuk, V. M., Doktorovych, I. V., Yuryev, G. V., Fodchuk, I. M., Chorok, Ye. O. (2018). Additional errors occurring in illuminators. Herald of Khmelnytskyi national university, 3 (261), 253-257. Available at: http://journals.khnu.km.ua/vestnik/pdf/ tech/pdfbase/2018/2018_3/jrn/pdf/43.pdf

7. Tankevych, Ye. M., Yakovlieva, I. V., Varskyi, G. M. (2016). Increasing the Accuracy of Voltage Measuring Channels of Electrical Power Object Control Systems. Visnyk Vinnytskoho politekhnichnoho instytutu, 1, 79-84. Available at: https://visnyk.vntu.edu. ua/index.php/visnyk/article/download/1880/1880/

8. Stepaniak, M. M., Skalskyi, V. R., Stepaniak, M. V. (2010). Doslidzhennia mozhlyvosti pidvyshchennia tochnosti vymiriuvannia temperatury obertovykh obiektiv. Visnyk Natsionalnoho universytetu "Lvivska politekhnika", 686, 13-23. Available at: http:// ena.lp.edu.ua:8080/bitstream/ntb/34026/1/02.pdf

9. Yanenko, O. P., Mikhailenko, S. V., Lisnichuk, A. S. (2014). Radiometric modulation measuring device of intensity of optical radiation. Visnyk Natsionalnoho tekhnichnoho universytetu Ukrainy "Kyivskyi politekhnichnyi instytut". Ser.: Radiotekhnika. Radioaparatobuduvannia, 56, 96-101. Available at: http://nbuv.gov.ua/UJRN/VKPI_rr_2014_56_11

10. Wu, J., Chen, Y., Gao, S., Li, Y., Wu, Z. (2015). Improved measurement accuracy of spot position on an InGaAs quadrant detector. Applied Optics, 54 (27), 8049. doi: https://doi.org/10.1364/ao.54.008049

11. Hidalgo-López, J. A., Fernández-Ramos, R., Romero-Sánchez, J., Martín-Canales, J. F., Ríos-Gómez, F. J. (2018). Improving Accuracy in the Readout of Resistive Sensor Arrays. Journal of Sensors, 2018, 1-12. doi: https://doi.org/10.1155/2018/9735741

12. Orozco, L. (2011). Optimizing Precision Photodiode Sensor Circuit Design. Analog Devices. Available at: https://www.analog. com/media/en/technical-documentation/tech-articles/Optimizing-Precision-Photodiode-Sensor-Circuit-Design-MS-2624.pdf

13. Zhang, J., Qian, W., Gu, G., Mao, C., Ren, K., Wu, C. et. al. (2019). Improved algorithm for expanding the measurement linear range of a four-quadrant detector. Applied Optics, 58 (28), 7741. doi: https://doi.org/10.1364/ao.58.007741

14. Hobbs, M. J., Tan, C. H. and Willmott, J. R. (2013). Evaluation of phase sensitive Hobbs, M. J., Tan, C. H., Willmott, J. R. (2013). Evaluation of phase sensitive detection method and $\mathrm{Si}$ avalanche photodiode for radiation thermometry. Journal of Instrumentation, 8 (03), P03016-P03016. doi: https://doi.org/10.1088/1748-0221/8/03/p03016

15. Petrovska, G., Demkovych, I. (2010). Apparatus for measurement absorption of the thin-film coatings by the photothermal method. Electrical Engineering, 61, 128-134. Available at: http://elit.lnu.edu.ua/pdf/61_17.pdf

16. Riazimehr, S., Kataria, S., Bornemann, R., Haring Bolívar, P., Ruiz, F. J. G., Engström, O. et. al. (2017). High Photocurrent in Gated Graphene-Silicon Hybrid Photodiodes. ACS Photonics, 4 (6), 1506-1514. doi: https://doi.org/10.1021/acsphotonics.7b00285

17. Lewis, G., Merken, P., Vandewal, M. (2018). Enhanced Accuracy of CMOS Smart Temperature Sensors by Nonlinear Curvature Correction. Sensors, 18 (12), 4087. doi: https://doi.org/10.3390/s18124087

18. Qin, J., Cui, S., Dai, J. (2020). Noise Analysis and Compensation Strategy of Photoelectric Detection Circuit. Journal of Physics: Conference Series, 1601, 022047. doi: https://doi.org/10.1088/1742-6596/1601/2/022047

19. Chen, C.-C., Chen, C.-L., Lin, Y. (2016). All-Digital Time-Domain CMOS Smart Temperature Sensor with On-Chip Linearity Enhancement. Sensors, 16 (2), 176. doi: https://doi.org/10.3390/s16020176

20. Shcherban', V., Korogod, G., Kolysko, O., Kolysko, M., Shcherban', Y., Shchutska, G. (2020). Computer simulation of multiple measurements of logarithmic transformation function by two approaches. Eastern-European Journal of Enterprise Technologies, 6 (4 (108)), 6-13. doi: https://doi.org/10.15587/1729-4061.2020.218517 
21. Shcherban, V., Korogod, G., Chaban, V., Kolysko, O., Shcherban', Y., Shchutska, G. (2019). Computer simulation methods of redundant measurements with the nonlinear transformation function. Eastern-European Journal of Enterprise Technologies, 2 (5 (98)), 16-22. doi: https://doi.org/10.15587/1729-4061.2019.160830

22. Soboleva, N. A., Melamid, A. E. (1974). Fotoelektronnye pribory. Moscow: «Vysshaya shkola», 376. Available at: https:// lib.convdocs.org/docs/index-20291.html

23. Kondratov,V.T.(2010). Metody izbytochnyhizmereniy:osnovnyeopredeleniyaiklassifikatsiya. Visnyk Khmelnytskohonatsionalnoho universytetu. Tekhnichni nauky, 3, 220-232. Available at: http://journals.khnu.km.ua/vestnik/pdf/tech/2010_3/47kon.pdf

24. Kondratov, V. T. (2015). The theory redundant and super-redundant measurements: super-redundant measurements of resistance of resistors and resistive sensors. The message 1. Vymiriuvalna ta obchysliuvalna tekhnika v tekhnolohichnykh protsesakh, 4, 7-22. Available at: http://nbuv.gov.ua/UJRN/vott_2015_4_3

25. Kondratov, V. T. (2009). Teoriya izbytochnyh izmereniy: universal'noe uravnenie izmereniy. Visnik Hmel'nits'kogo natsional'nogo universitetu. Tekhnichni nauky, 5, 116-129. Available at: http://journals.khnu.km.ua/vestnik/pdf/tech/2009 5/zmist.files/23kon.pdf

A simulation model of a photoplethysmographic signal under psychoemotional stress taking into account the nature of signals of biological origin and stress response stages was developed. The method of constructing the simulation model is based on reconstructing the waveform and coding points of the signal taking into account the stress response curve using harmonic functions at characteristic time intervals. Using the simulation model of the photoplethysmographic signal under psychoemotional stress with previously known parameters allows validation of methods and algorithms for processing such data. It was found that in the process of simulation, it is necessary to take into account the signal frequency, random component and stress response curve. This complicates the simulation algorithm. However, using the simulation model with variable input parameters allows reproducing the signal with an emphasis on stress response stages. One of the features of the proposed model is the ability to reproduce the signal by coding points for amplitude and time intervals using harmonic functions. The relative error for the amplitude variation of the model and experimental data is $3.97 \%$, and for the period - $3.41 \%$. Calculation of Student's t-test showed a statistically insignificant difference: $p=0.296$ for the amplitude and $p=0.275$ for the peri$o d$. This indicates that the simulation model takes into account the signal characteristics under stress: frequency, random component and stress response curve. Using the proposed simulation model is an adequate way to assess methods and algorithms for analyzing the state of the cardiovascular system under psychoemotional stress

Keywords: harmonic function, simulation model, periodic signal, psychoemotional stress, photoplethysmographic signal

\title{
DEVELOPMENT OF A SIMULATION MODEL OF A PHOTOPLETHYSMOGRAPHIC SIGNAL UNDER PSYCHOEMOTIONAL STRESS
}

\author{
Evhenia Yavorska \\ PhD, Associate Professor, Head of Department* \\ E-mail: yavorska_eb@yahoo.com \\ Oksana Strembitska \\ Postgraduate Student* \\ E-mail: oksanaoliinyk93@gmail.com \\ Michael Strembitskyi \\ $\mathrm{PhD}$, Associate Professor \\ Department of Instruments and \\ Control-Measurement Systems** \\ E-mail: m.strembitskyy@gmail.com \\ Iryna Pankiv \\ Postgraduate Student* \\ E-mail: Ira_Pankiv@ukr.net \\ *Department of Biotechnical Systems** \\ **Ternopil Ivan Puluj National Technical University \\ Ruska str., 56, Ternopil, Ukraine, 46001
}

Received date 23.02.2021

Accepted date 12.04.2021

Published date 19.04.2021
How to Cite: Yavorska, E., Strembitska, O., Strembitskyi, M., Pankiv, I. (2021). Development of a simulation model of a photoplethysmographic signal under psychoemotional stress. Eastern-European Journal of Enterprise Technologies, 2 (9 (110)), 36-45. doi: https://doi.org/10.15587/1729-4061.2021.227001

\section{Introduction}

The occurrence of psychoemotional stress at the dentist is a factor in the development and progression of diseases of the cardiovascular system (CVS) - hypertensive crisis, myocardial infarction, stroke, arrhythmia, etc. [1]. According to testing data, $80 \%$ of patients who seek help from a dentist suffer from dentophobia [2,3]. Providing dental care to the population belongs to the mass types of medical care, as $20-25 \%$ appeals are related to oral diseases (345-550 cases per 1,000 inhabitants) [4]. Timely detection of CVS disorders due to psychoemotional stress at the dentist is an urgent medical problem. One of the non-invasive methods to collect data on the state of the CVS, including under psychoemotional stress, is determining pulse signal parameters - photoplethysmography [5, 6]. Since the analysis of the photoplethysmographic signal is 